\section{Clinical evaluation of Morgellons disease in a cohort of North American patients}

\author{
Melissa C. Fesler, ${ }^{1}$ \\ Marianne J. Middelveen, ${ }^{2}$ \\ Raphael B. Stricker ${ }^{1}$
}

${ }^{1}$ Union Square Medical Associates, San

Francisco, CA, USA; ${ }^{2}$ Atkins Veterinary

Services, Calgary, AB, Canada

\begin{abstract}
Morgellons disease (MD) is a dermatological condition characterized by aberrant production of keratin and collagen fibers in skin. Although infection with Borrelia burgdorferi, the causative agent of Lyme disease (LD), has been associated with MD, relatively few studies have hitherto provided epidemiological evidence regarding this association. A cohort of 1000 seropositive North American LD patients was evaluated for the presence of MD. Patients were diagnosed with MD based on detection of microscopic fibers in skin lesions or under unbroken skin. Demographic and clinical features of MD patients were analyzed, and laboratory testing for tickborne coinfections and other infectious agents, was performed. Subjective and objective features of MD were analyzed using statistical methods. Of 1000 seropositive LD patients, $60(6 \%)$ were diagnosed with MD. Of these 60 patients, $75 \%$ were female and $78 \%$ presented in the late disseminated stage of MD. All 60 patients $(100 \%)$ were seropositive for $B$. burgdorferi infection. Tickborne coinfections in these patients included Babesia spp (62\%), Bartonella and Rickettsia (25\% each), Ehrlichia (15\%) and Anaplasma (10\%). Helicobacter pylori was detected in $12 \%$ of MD patients. In all, $77 \%$ of MD patients had one or more coinfections. This study confirms recent findings that MD occurs in a limited subset of LD patients. The clinical and genetic determinants of $\mathrm{MD}$ in $\mathrm{LD}$ patients require further study.
\end{abstract}

\section{Introduction}

Morgellons disease (MD) is a dermatological condition characterized clinically by the presence of multicolored microscopic fibers in skin lesions or lying under unbroken skin. ${ }^{1-7}$ The disease is associated with overproduction of keratin and collagen in cutaneous tissue. Historically, MD has been misclassified as a delusional disorder due to its sometimes bizarre symptomatology in patients who present with claims of colorful fibers or parasites protruding from or crawling within the dermis. In contrast, recent peer reviewed publications demonstrate that MD is a true somatic illness associated with Borrelia infection, and not a delusional disorder. $^{1-7}$ Other studies have shown that a similar dermopathy occurs in cattle and dogs, providing further evidence that MD is a dermatological condition associated with spirochetal infection..$^{8} 9$

The spirochete Borrelia burgdorferi is the etiologic agent of Lyme disease (LD). According to the Centers for Disease Control and Prevention (CDC), there are more than 300,000 newly diagnosed cases of LD each year in the USA, ${ }^{10}$ making it the fastest growing epidemic of our time. ${ }^{11} \mathrm{LD}$ is twice as common as breast cancer, ${ }^{12}$ six times more common than HIV/AIDS, ${ }^{13} 20$ times more common than hepatitis $\mathrm{C}$ virus (HCV) infection, ${ }^{14}$ and 30 times more common than tuberculosis in the USA. ${ }^{15}$ Unfortunately, a large number of LD cases go either undiagnosed or misdiagnosed, resulting in chronic infection and potential complications associated with the tickborne disease, including MD. ${ }^{6,16}$

Although previous studies have shown a strong clinical association between LD and MD, ${ }^{2,3,5-7}$ few studies have provided epidemiological data for MD. Recently, Mayne evaluated a cohort of Australian patients and found that $6 \%$ of seropositive Lyme patients were positive for MD based on skin microscopy. ${ }^{17}$ This publication was the first to provide an epidemiological assessment of MD as related to LD. To date, there are no similar studies regarding the prevalence of MD in North America. Other studies have implicated tickborne coinfections and Helicobacter pylori infection in the etiology of MD. ${ }^{18,19}$

The present study is the first to analyze the prevalence and clinical characteristics of MD in a cohort of North American patients with LD drawn from a single medical practice.

\section{Materials and Methods}

\section{Patients and data collection}

Patients were recruited from a medical practice located in San Francisco, CA, specializing in the diagnosis and treatment of tickborne diseases. All patients were residents of North America. Informed consent for data collection was obtained from each patient, and the anonymous data collection protocol was approved by the Western
Correspondence: Raphael B. Stricker, 450 Sutter Street, Suite 1504, San Francisco, CA 94108 USA.

Tel.: 415.399.1035 - Fax: 415.399.1057.

E-mail: rstricker@usmamed.com

Key words: Morgellons disease; Lyme disease; Borrelia burgdorferi; Spirochetes; Dermopathy; Borrelial dermatitis.

Acknowledgements: the authors thank our patients for participating in the study. We also thank Dr. Jae Brodsky for statistical analysis.

Funding: supported in part by a grant from the Lindorf Family Foundation, Newark, OH, USA.

Conflict of interests: the authors declare no conflict of interests.

Received for publication: 27 February 2018. Accepted for publication: 16 March 2018.

This work is licensed under a Creative Commons Attribution-NonCommercial 4.0 International License (CC BY-NC 4.0).

(C) Copyright M.C. Fesler et al., 2018

Licensee PAGEPress, Italy

Dermatology Reports 2018; 10:7660 doi:10.4081/dr.2018.7660

Institutional Review Board (WIRB), Puyallup, WA. Patients were included in the study if they met the diagnostic criteria for MD through identification of fibers visible in skin lesions or under unbroken skin using a hand-held microscope, as previously described. ${ }^{1-6}$ MD classification based on the duration of disease (early versus late) and the extent of skin involvement (localized versus disseminated) was performed as previously described. ${ }^{5}$

All subjects were required to have LD testing and tickborne coinfection testing through a single laboratory, and they were required to have repeat testing to confirm the diagnosis of LD. Prior antibiotic treatment was not an excluding factor. Subjective data including a thorough history was obtained during the initial patient encounter. Objective data including physical findings and laboratory testing was collected during each patient visit and through various laboratories.

\section{Laboratory assessment}

Testing for tickborne diseases was performed through IGeneX Laboratory in Palo Alto, CA. IGeneX is a high-complexity testing laboratory that has Clinical Laboratory Improvement Amendments (CLIA) certification. Seropositivity for LD and tickborne coinfections was based on 
standard laboratory interpretation criteria, as previously described. ${ }^{20}$ Serological testing for Helicobacter pylori infection was performed through various laboratories using standard assays. ${ }^{21}$

To evaluate companion diagnostic features of tickborne diseases, CD57 natural killer (NK) cell subset testing was performed by Bioreference Laboratories (Elmwood Park, NJ) or LabCorp, Inc. (Burlington, NC). ${ }^{22}$ Testing for complement protein $\mathrm{C} 4 \mathrm{a}$, a marker of inflammation, was performed by National Jewish Health Laboratory in Denver, CO. ${ }^{23}$ Low CD57 NK cells and elevated C4a levels are common in LD patients. ${ }^{22,23}$

Statistical analysis was performed using $\mathrm{R}$ statistical software version 3.2.5 (R Foundation for Statistical Computing, Vienna, Austria). The Student t-test was used for parametric variables and the Pearson correlation coefficient $r$ was used for non-parametric variables.

\section{Results}

A total of 1000 patients (665 females and 335 males) who were residents of North America and seropositive for LD were

Table 1. Sex of MD patients. MD was more common in women with a 3:1 female-tomale ratio $(P=0.001)$.

\begin{tabular}{lcc} 
Sex & Number (n) & Percent (\%) \\
Female & 45 & 75 \\
Male & 15 & 25 \\
\hline
\end{tabular}

Table 2. Clinical classification of MD patients based on Reference 5 .

\begin{tabular}{lcc} 
MD & $\begin{array}{c}\text { Number } \\
\text { Classification }\end{array}$ & $\begin{array}{c}\text { Percent } \\
(\%)\end{array}$ \\
Early localized MD & 5 & 8 \\
Early disseminated MD & 4 & 7 \\
\hline Late localized MD & 4 & 7 \\
Late disseminated MD & 47 & 78 \\
\hline
\end{tabular}

Table 3. Symptoms noted in MD patients.

\begin{tabular}{lcc} 
Symptoms & $\begin{array}{c}\text { Number } \\
\text { (n) }\end{array}$ & $\begin{array}{c}\text { Percent } \\
(\%)\end{array}$ \\
Musculoskeletal symptoms & 56 & 93 \\
Fatigue & 53 & 88 \\
\hline Insomnia & 48 & 80 \\
Cognitive impairment & 30 & 50 \\
\hline Depression & 30 & 50 \\
Hypothyroidism & 20 & 33 \\
\hline Anxiety & 20 & 33 \\
Neuropathy & 20 & 33 \\
\hline
\end{tabular}

screened for the study. Patients ranged in age from 22 months to 93 years. Of this total, 62 were diagnosed with MD. Two patients were excluded from the study. One subject never returned after the first visit and the other never received follow-up testing, although the subject tested positive for tickborne coinfections. A total of 60 patients were included for data analysis.

MD tended to be more common in women, with a 3:1 female-to-male ratio $(\mathrm{P}=0.001)$, as shown in Table 1 . The median age of MD patients was 52 years, with an age range of 26-75 years, as shown in
Figure 1, and there was no statistical difference in age between men and women $(\mathrm{P}=0.2)$, as shown in Figure 2. More than three-quarters of MD patients $(78 \%)$ were diagnosed with late disseminated MD at presentation, as shown in Table 2, and the most common patient symptoms are shown in Table 3. The three most common symptoms were musculoskeletal pain, fatigue and insomnia; the next most common were cognitive impairment and depression, followed by hypothyroidism, anxiety and neuropathy.

Coinfection testing to evaluate other

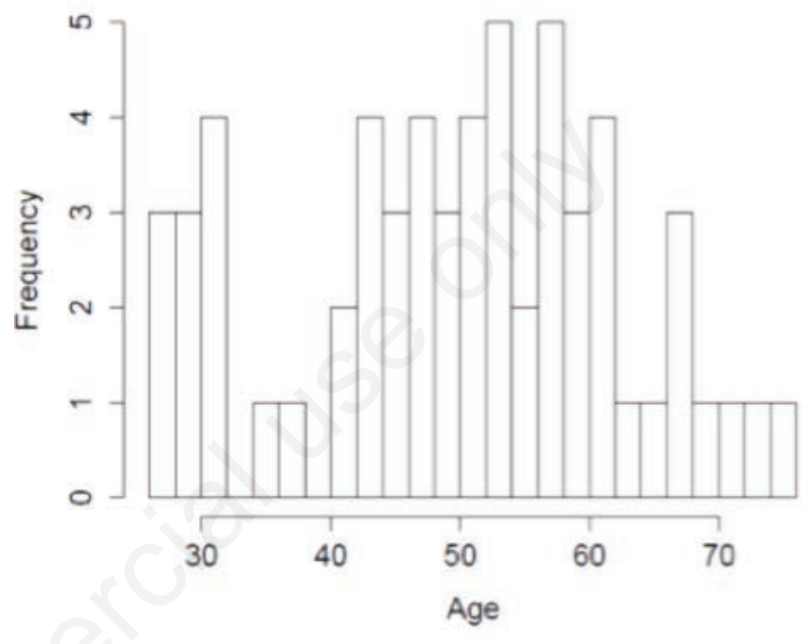

Figure 1. Age of MD patient cohort. Each bar represents a two-year span. Frequency represents the number of individuals who fall within the reported two-year span. The median age was 52 years with a range of 26 to 75 years.

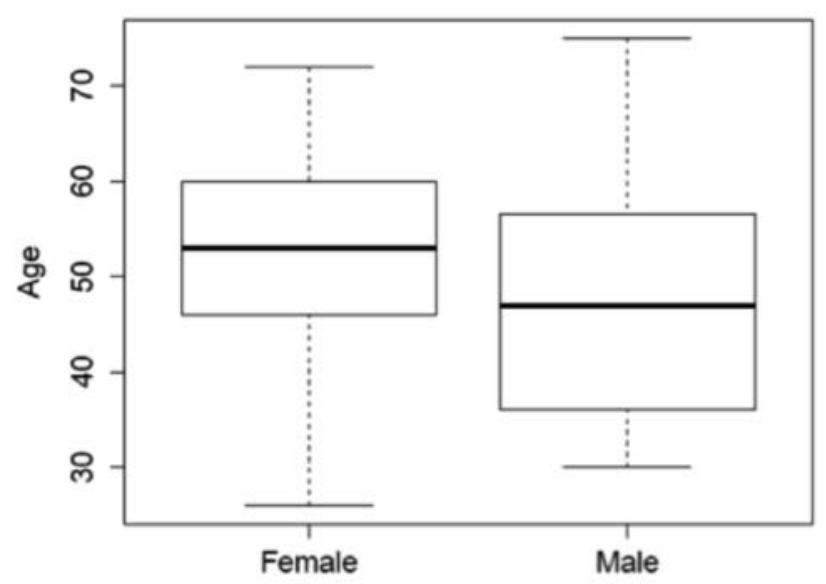

Figure 2. Sex versus age in MD patient cohort. Wings represent the age range for men and women. Each box represents the standard deviation for each sex. Bars represent the mean age for each sex. There was no statistical difference in age between men and women $(\mathbf{P}=\mathbf{0 . 2})$. The female patients (mean: $\mathbf{5 1 . 2}$ years) had a tendency to be slightly older than the male patients (47.9 years). The standard deviations of the two groups were similar (12.2 years and 14.4 years) and the shape of their distributions were of similar symmetry. A two-sample t-test with an alternative hypothesis of the male mean less than the female mean had a t-statistic of 0.7942 and P-value of 0.218 ( 21 degrees of freedom). 
infectious agents in the etiology of MD was performed on all patients, and the documented coinfections are listed in Table 4. In our 60 MD patients, 30 (50\%) were seropositive for Babesia duncani, 7 (12\%) for Babesia microti, 15 (25\%) for Bartonella henselae, 15 (25\%) for Rickettsia spp., 9 (15\%) for Ehrlichia, 6 (10\%) for Anaplasma, and 7 (12\%) were seropositive for Helicobacter pylori. While $23 \%$ of MD patients had no coinfection, $33 \%$ had a single coinfection and $44 \%$ had multiple coinfections (Table 5). Examination of companion diagnostic tickborne disease testing in the MD cohort revealed that $66 \%$ had decreased CD $57 \mathrm{NK}$ cells and 33\% had increased C4a levels. A lower CD57 NK level correlated with a higher $\mathrm{C} 4 \mathrm{a}$ inflammatory marker level in a non-linear manner $(\mathrm{r}=0.02)$, as shown in Figure 3.

\section{Discussion}

According to the CDC, approximately 329,000 cases of LD are diagnosed each year in the USA (10). In our cohort of North American LD patients, we found that $6 \%$ had MD (Table 1). Based on these numbers, there may be at least 19,740 annual cases of $\mathrm{MD}$ in the USA (MD prevalence $=6 \%$; MD incidence $=19,740$ ). Considering cumulative rates of LD over the past 20 years, there may be approximately 5,304,500 total cases

Table 4. Variety of coinfections found in MD patients.

\begin{tabular}{lcc} 
Infection & $\begin{array}{c}\text { Number } \\
\text { (n) }\end{array}$ & $\begin{array}{c}\text { Percent } \\
(\%)\end{array}$ \\
\hline Borrelia burgdorferi & 60 & 100 \\
Babesia duncani & 30 & 50 \\
\hline Babesia microti & 7 & 12 \\
Bartonella henselae & 15 & 25 \\
\hline Rickettsia spp. & 15 & 25 \\
Ehrlichia & 9 & 15 \\
\hline Anaplasma & 6 & 10 \\
Helicobacter pylori & 7 & 12 \\
\hline
\end{tabular}

Table 5. Number of coinfections in MD patients.

\begin{tabular}{lcc} 
Coinfections & $\begin{array}{c}\text { Number } \\
\text { (n) }\end{array}$ & $\begin{array}{c}\text { Percent } \\
\text { (\%) }\end{array}$ \\
\hline 0 & 14 & 23 \\
1 & 20 & 33 \\
\hline 2 & 18 & 30 \\
3 & 4 & 7 \\
\hline 4 & 4 & 7 \\
\hline
\end{tabular}

of LD in the USA, corresponding to a total of at least 318,270 cases of MD. At this rate, and as a reference, MD is slightly more common than HCV infection, 1.5 times more common than pediatric cancer and almost three times more common than amyotrophic lateral sclerosis in the USA. ${ }^{14,24,25}$

Patients were more likely to present with late disseminated MD in our cohort (Table 2), and systemic symptoms that often accompany LD were common (Table 3). Many of these symptoms tended to precede the onset of MD skin lesions. Tickborne coinfections including Babesia spp (62\%), Bartonella and Rickettsia (25\% each), Ehrlichia (15\%) and Anaplasma (10\%) were found in patients with MD (Table 4), and Helicobacter pylori infection was detected in $12 \%$ of MD patients. More than three-quarters of patients had at least one coinfection (Table 5), and it is unclear if these coinfections serve as a trigger or exacerbate the condition. Studies have found Helicobacter pylori and Bartonella in tissue biopsies of MD patients, ${ }^{18,19}$ and further studies are needed to investigate the role of coinfections in MD severity and presentation. Our study confirms that there is a greater tendency for women to develop $\mathrm{MD}$, and the 3:1 female predominance exceeds the 2:1 female predominance of LD in our cohort. ${ }^{2,26}$ Savely and Stricker ${ }^{2}$ previously showed that women are more likely to develop this dermopathy in conjunction with $\mathrm{LD}$, reflecting the fact that certain disease processes may be recognized more fre- quently in women due to an exaggerated response to infection. ${ }^{26}$ Women also tend to be more meticulous with dermatologic care, and increased awareness of skin changes could account for a female predominance of the disease. Genetic factors may also play a role in MD. Preliminary genetic studies have demonstrated nine genes with significant sequence variation in MD patients (Sapi E, University of New Haven, unpublished observation 2017). There was no difference in age at presentation between men and women, although men tended to present at a slightly younger age (Figures 1 and 2).

As noted above, patients were more likely to present with late disseminated MD (Table 2). Since MD apparently represents a dermatopathological spectrum associated with $\mathrm{LD}$, it is possible that many individuals do not realize they have the dermatological condition until later in its course, when more symptoms are present and/or more skin lesions appear. As in the case of LD, few practitioners diagnose and treat MD, and patients may have a difficult time obtaining treatment during the early stages of the disease. ${ }^{27}$ Consequently, there may be a greater tendency to seek medical care only after the disease worsens.

Future studies are required to evaluate and confirm MD epidemiological data obtained in this study. Larger sample sizes should be examined and direct methodology such as culture, immunohistochemistry and molecular testing, should be performed to confirm the tickborne disease etiology of 
MD. Furthermore, genetic susceptibility for MD should be evaluated, and additional animal studies in dogs, cattle and other models should be conducted to determine the prevalence of MD in association with spirochetal infection and genetic factors in animal and human populations.

\section{Conclusions}

To our knowledge, this is the first study that documents the prevalence and clinical characteristics of MD in North American patients. The study confirms recent findings that MD occurs in a limited subset of LD patients, and other tickborne coinfections were inconsistently associated with MD. The clinical and genetic determinants of $\mathrm{MD}$ in LD patients require further study.

\section{References}

1. Savely VR, Leitao MM, Stricker RB. The mystery of Morgellons disease: infection or delusion. Am J Clin Dermatol 2006;7:1-5.

2. Savely VR, Stricker RB. Morgellons disease: Analysis of a population with clinically confirmed microscopic subcutaneous fibers of unknown etiology. Clin Cosmet Investig Dermatol 2010;3:67-78.

3. Middelveen MJ, Burugu D, Poruri A, et. al. Association of spirochetal infection with Morgellons disease. F1000Res 2013;2:25.

4. Harvey WT, Bransfield RC, Mercer DE, et al. Morgellons disease, illuminating an undefined illness: a case series. J Med Case Rep 2009;3:8243.

5. Middelveen MJ, Bandoski C, Burke J, et. al. Exploring the association between Morgellons disease and Lyme disease: identification of Borrelia burgdorferi in Morgellons disease patients. BMC Dermatol 2015;15:1.

6. Middelveen MJ, Stricker RB. Morgellons disease: a filamentous borrelial dermatitis. Int $\mathrm{J}$ Gen Med 2016;9:349-54.

7. Middelveen MJ, Fesler MC, Stricker
RB. History of Morgellons disease: from delusion to definition. Clin Cosmet Investig Dermatol 2018;11:7190.

8. Middelveen MJ, Stricker RB. Filament formation associated with spirochetal infection: a comparative approach to Morgellons disease. Clin Cosmet Investig Dermatol 2011;4:167-77.

9. Middelveen MJ, Rotaru GM, McMurray JL, et al. Canine filamentous dermatitis associated with Borrelia infection. J Vet Sci Med Diagn 2016;5:6.

10. CDC. How many people get Lyme disease? Available from: https://www.cdc.gov/lyme/stats/humancases.html Accessed: March 30, 2017.

11. Stricker RB, Middelveen MJ. Sexual transmission of Lyme disease: challenging the tickborne disease paradigm. Expert Rev Anti Ther 2015;13:1303-6.

12. CDC. Breast cancer statistics. Available from: https://www.cdc.gov/ cancer/breast/statistics/ Accessed: March 30, 2017.

13. CDC. HIV in the United States: at a glance. Available from: https:/www.cdc.gov/hiv/statistics/over view/ataglance.html Accessed: March 30, 2017.

14. CDC. Viral hepatitis: Statistics \& surveillance. Available from: https://www.cdc.gov/hepatitis/statistics/. Accessed: March 30, 2017.

15. CDC. Tuberculosis (TB). Available from: https://www.cdc.gov/tb/statistics/ Accessed: March 30, 2017.

16. Stricker RB, Johnson L. Lyme disease: the promise of Big Data, companion diagnostics and precision medicine. Infect Drug Resist 2016;9:215-9.

17. Mayne PJ. Clinical determinants of Lyme borreliosis, babesiosis, bartonellosis, anaplasmosis and ehrlichiosis in an Australian cohort. Int $\mathrm{J}$ Gen Med 2014;8:15.

18. Bandoski C. Evidence for the presence of human pathogens Borrelia and Helicobacter in Morgellons patients' skin samples. Presented at: 7th Annual Medical-Scientific Conference on Morgellons Disease; March 29-30, 2014; Austin, TX. Available from: http://www.thecehf. org/cheryl-bandos- ki.html Accessed: September 30, 2016.

19. Allen L, Saylor-Hefley C. Morgellons under investigation: identification of associated microorganisms by molecular analysis of epithelial samples. Presented at: 7th Annual MedicalScientific Conference on Morgellons Disease; March 29-30, 2014; Austin, TX. Available from: http://www.thecehf.org/resources/OSU\%20_2015\%20_ Research.pdf Accessed: September 30, 2016.

20. Shah JS, Du Cruz I, Narciso W, Lo W, Harris NS. Improved sensitivity of Lyme disease Western blots prepared with a mixture of Borrelia burgdorferi strains 297 and B31. Chronic Dis Int 2014;1:7.

21. She RC, Wilson AR, Litwin CM. Evaluation of Helicobacter pylori immunoglobulin G (IgG), IgA, and IgM serologic testing compared to stool antigen testing. Clin Vaccine Immunol 2009;16:1253-5.

22. Stricker RB, Winger EE. Decreased CD57 lymphocyte subset in patients with chronic Lyme disease. Immunol Lett 2001;76:43-8.

23. Stricker RB, Savely VR, Motanya NC, Giclas PC. Complement split products $\mathrm{C} 3 \mathrm{a}$ and $\mathrm{C} 4 \mathrm{a}$ in chronic Lyme disease. Scand J Immunol 2009;69:64-9.

24. National Cancer Institute. Childhood cancers. Available from: https://www.cancer.gov/types/childhood-cancers Accessed: October 30, 2017.

25. CDC. Prevalence of amyotrophic lateral sclerosis — United States, 2012-2013. Surveillance Summaries 2016; 65:1-12. Available from: https://www.cdc.gov/mmwr/volumes/6 5/ss/ss6508a1.htm. Accessed October 30, 2017.

26. Stricker RB, Johnson L. Gender bias in chronic Lyme disease. J Womens Health (Larchmt). 2009;18:1717-8.

27. Johnson L, Aylward A, Stricker RB. Health care access and burden of care for patients with Lyme disease: a large United States survey. Health Policy. 2011;102:64-71. 